\title{
Development of optoelectronic-based pulsed current sensor to detect the poor laser discharge condition
}

\author{
ANIL S NAYAK*, RAJASREE VIJAYAN, DEEPAK NAPHADE, V S RAWAT and \\ K G MANOHAR
}

Laser and Plasma Technology Division, Bhabha Atomic Research Centre, Mumbai 400 085, India

e-mail: anilsn@barc.gov.in

MS received 30 September 2016; revised 27 December 2016; accepted 19 February 2017; published online 3 August 2017

\begin{abstract}
A current transformer (CT)-based sensor has been developed to detect poor discharge conditions in copper vapour laser. The optoelectronic-based pulsed current sensor architecture involves the optical transmitter-receiver HFBR, high-frequency current transformer, and fiber optic link. The CT has been designed and calibrated to ensure generation of an optical signal at the current threshold crossover. Bandwidth analysis of the CT is carried out using the bode plot. The optoelectronic inter-conversion of the pulsed voltage of the CT and transmission via fiber optic link provides the non-contact current sensing and remote signal processing of the signal. This study discusses the details of the sensor.
\end{abstract}

Keywords. High-frequency current transformer; HFBR; copper vapour laser.

\section{Introduction}

Copper vapour laser (CVL) is a pulsed electric discharge pumped laser. The electrical excitation of the CVL takes place at a pulsed voltage of $10-15 \mathrm{kV}$ at $9 \mathrm{kHz}$ pulse repetition frequency. The inductor of $60 \mu \mathrm{H}$ is connected in parallel with the laser head. The windings of the inductor are wound on the non-magnetic Teflon core. The inductor is designed such that it offers very high impedance as compared to the laser head discharge impedance. As shown in figure 1, the energy delivered by the pulsed excitation circuit results in current flowing through the laser head $\left(I_{1}\right)$ and the inductor $\left(I_{2}\right)$. During proper discharge between the CVL electrodes, the discharge current is much greater than the inductor current $\left(I_{1} \gg I_{2}\right)$. The discharge condition, however, becomes poor under two different conditions. First, the vacuum integrity of the laser head is not intact. Owing to a leak in the vacuum system, the atmospheric gases enter into the CVL, causing increase in breakdown voltage requirement. Since the electrical excitation system is not designed to operate under such abnormal increase in the voltage requirements, either there is no discharge or the discharge is in the laser head. Second, the absence of discharge between electrodes is also due to the erosion of the electrode pins. Under both the circumstances, the electrical energy flows substantially through the bypass inductor connected in parallel to the laser head (i.e. $I_{1} \ll I_{2}$ ). The joules heating due to current flowing through the windings resistance increases the temperature of

*For correspondence the winding as well as the core leading to an alarming condition. A sensor was developed to detect this condition, which could sense poor discharge and raise an alarm for the operator to initiate safety measures.

Current sensing technology has become an important element for protection and control in the engineering systems. The overview, advantages and limitations of the current sensing technology have been discussed by Xiao et al [1]. Different current sensing elements such as current shunt, Rogowski coil, current transformer and hall-effect-based sensors are used. The current shunt offers non-isolated current measurement, while the Hall-effect sensor, self-integration and active integration-based Rogowski coil and current transformer facilitates isolated current measurement [2-4].

The wide bandwidth high-frequency CT (HFCT) utilises $\mathrm{Ni}-\mathrm{Zn}$ or $\mathrm{Mn}-\mathrm{Zn}$ ferrite core, depending on the frequency of application. The coupling coefficient between the primary and the secondary is close to unity because of high permeability. The technology of isolating low voltage subsystems of current sensor from high voltage has been implemented using optical fiber for power metering $[5,6]$. The current transformer samples the bus-bar current at high voltage and converts it into small current; then the small current is converted into digital signals, and further transmitted through optical fiber to the lower voltage side and processed therein. The digital circuitry to convert the sampled current to digital signal is placed in the vicinity of the high-voltage bus bar and the DC supply required for the functioning of the same circuitry is derived from the busbar supply using the concept of dual-power supply [5]. In 


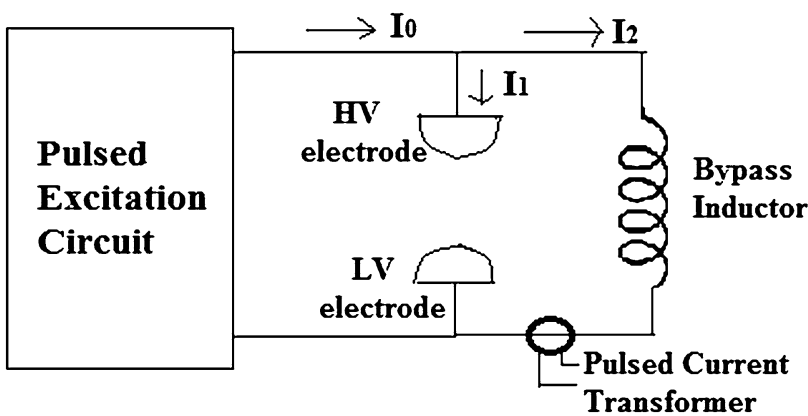

Figure 1. System schematic (pulsed excitation circuit, laser head and bypass inductor). The pulsed current transformer is connected in the return path of inductor.

this paper, the sampled signal is passively converted into the optical signal using an HFBR transmitter without using any active power supplies or from the supply derived from the source, thus making it immune to the EMI environment generated due to the fast rise of time current pulses.

\section{Pulsed current sensor architecture and principle}

The schematic of the optoelectronics-based pulsed current sensor is shown in figure 2. The wide bandwidth current transformer is terminated with the burden resistance $R_{1}$. The voltage developed across the burden is itself used to drive the optical transmitter on considering proper polarity of the signal. When the voltage developed across $R_{1}$ reaches to the on-state voltage of the HFBR transmitter, the optical signal of the on-time duration equal to the base-tobase pulse width of the current signal is transmitted through the fiber optic link. The optical to electrical signal conversion takes place at the receiver end using the optical receiver HFBR connected in the external pull-up mode. The TTL signal thus generated is used to generate the alarm.

\subsection{Design of current transformer}

The current transformers are based on the magnetic coupling principle. It consists of a toroidal ferromagnetic core, on which a copper wire of $n$ turns is wound. This winding is terminated with a low-inductance burden resistor $R_{1}$. The conductor passing through the centre of the toroid carrying the time-varying current acts as the primary of the current transformer. The winding on the toroid acts as the secondary of the current transformer. The burden resistance is selected depending on the sensitivity required. For better performance, current transformer cores with high resistivity, low hysteresis, small eddy current losses and wide bandwidth are desired. The primary pulse current passing through the centre of the current transformer has a rise time of $200 \mathrm{~ns}$. The current sensor should have a wide bandwidth in order to achieve high fidelity of the actual current pulse signal. To

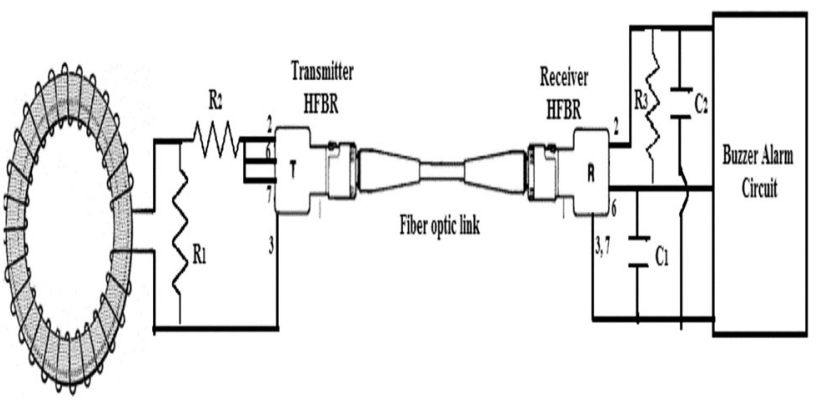

Figure 2. The schematic of optoelectronic-based pulsed current sensor.

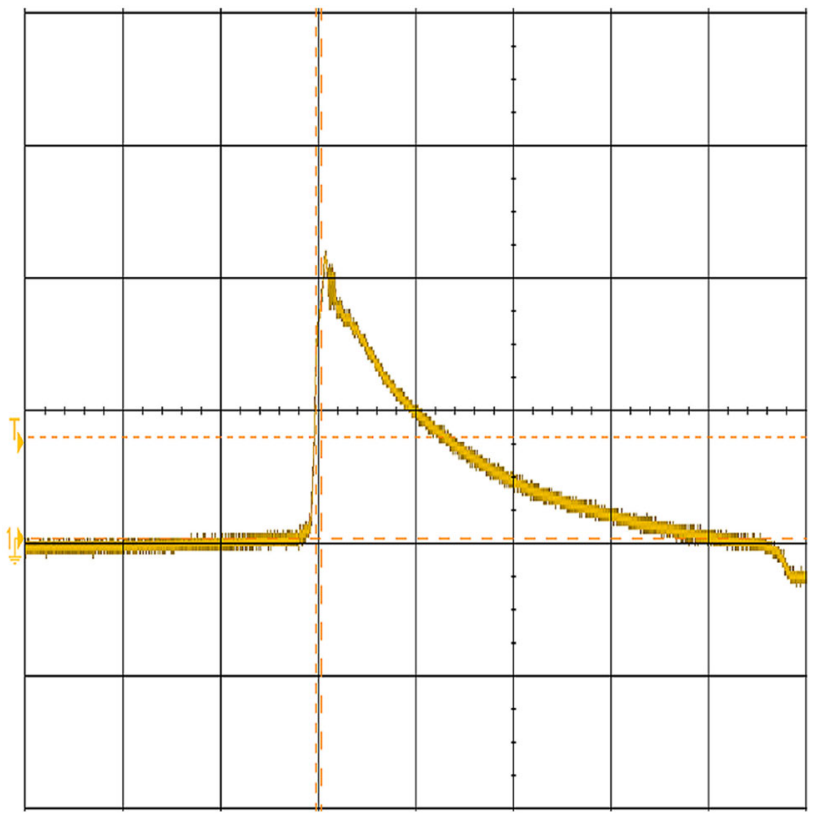

Figure 3. Typical current waveform through the inductor during good CVL discharge condition. Scale: $\mathrm{X}$ axis $-2 \mu \mathrm{s} / \mathrm{div}$; $\mathrm{Y}$ axis 2 V/div.

obtain a low value for the lower cut-off frequency, cores with high permeability are needed, while to increase the higher cut-off frequency, the leakage inductance and the parasitic capacitances should be minimised [7].

The technology used to develop this sensor depends on the conduction of the transmitter HFBR when the primary current increases beyond the threshold value. The threshold value depends on the current flowing through the bypass inductor, which is an indication of the discharge condition of the CVL. Figure 3 shows the waveform of the bypass inductor current during good CVL discharge condition. The current was measured using Pearson current monitor model 110 make having a sensitivity of $0.1 \mathrm{~V} / \mathrm{A}$. The peak current flowing through the inductor during good laser discharge condition is in the range of 25-30 A. Figure 4 shows the waveform of the bypass inductor current during bad CVL discharge condition. The peak current flowing through the inductor during bad laser 


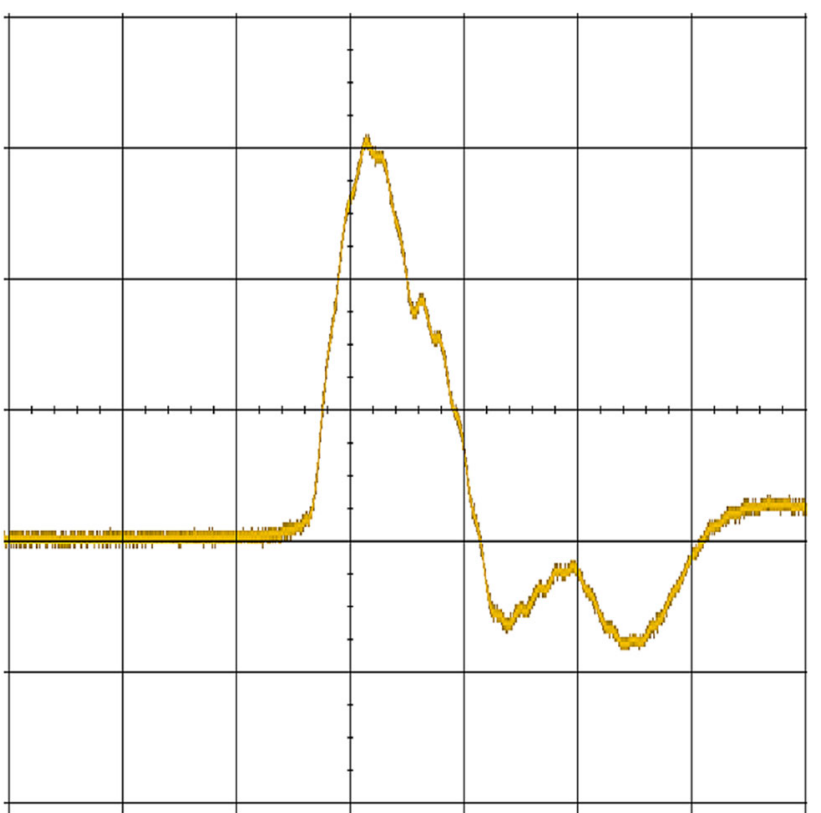

Figure 4. Typical current waveform through the inductor during bad CVL discharge condition. Scale: $\mathrm{X}$ axis $-1 \mu \mathrm{s} / \mathrm{div}$; $\mathrm{Y}$ axis $2 \mathrm{~V} / \mathrm{div}$.

discharge condition is in the range of 55-60 A. The continuous flow of this current through the inductor then leads to an alarming condition as described earlier. Thus, the sensor was developed with the threshold current value of 45 A flowing through the inductor. This threshold gives adequate safety margin below the peak value of 55-60 A.

The sensitivity of the current transformer for this sensor depends on the on-state conduction voltage $\left(V_{\text {on }}\right)$ of the HFBR and the threshold value of the current at which the sensor should operate $\left(I_{\mathrm{t}}\right)$. The on-state conduction voltage of the HFBR transmitter is $1.6-1.7 \mathrm{~V}$. Thus, the CT has been designed with the sensitivity $\left(G=V_{\mathrm{on}} / I_{\mathrm{t}}\right)$ of 0.037 V/A. Ferrite toroidal core T6320-CF197 manufactured by Cosmo Ferrites was used. The parameters of the chosen core were as follows: Outer diameter (O.D.) $=63 \mathrm{~mm}$, internal diameter (I.D.) $=38 \mathrm{~mm}$, height (h) $=20 \mathrm{~mm}$, toroid effective length $\left(L_{\mathrm{e}}\right)=152.1 \mathrm{~mm}$, effective core cross-sectional area $(\mathrm{Ac})=244.7 \mathrm{~mm}^{2}$, $\mu_{\mathrm{r}}=7000$, core resistivity $\left(\rho_{\mathrm{c}}\right)=0.2 \Omega-\mathrm{m}$ and inductance factor $\left(A_{1}\right)=13,000 \mathrm{nH} / N^{2}$, where $N$ is the number of turns wound on the toroid. The number of turns in the secondary winding was chosen to be 35 . A burden resistor of $1.3 \Omega$ was chosen. The required sensitivity of the CT, that is, $0.037 \mathrm{~V} / \mathrm{A}$ can be attained by varying the secondary number of turns or the burden resistance or both. To ensure that the required sensitivity with higher accuracy of current transformation and lower parasitic capacitance was attained, the burden resistance was kept at a lower value and lesser number of secondary turns was used. The CT should respond equally to all the frequency components present in the pulsed current signal with fast rise time of $200 \mathrm{~ns}$. Bandwidth analysis is carried out considering the equivalent circuit model of the CT shown in figure 5 .

Figure 5 shows the equivalent circuit of the $1: n$ current transformer. The primary current $(i)$ referred to secondary $(i / n)$ is sensed across the burden resistor $R_{\mathrm{B}} . L_{m}$ and $R_{c}$ are the magnetising inductance and the core parallel equivalent resistance of the secondary, respectively. $L_{2}$ and $R_{2}$ are the leakage reactance and the winding resistance of the secondary coil, respectively. The parasitic components on the primary and the secondary sides are shown as $C_{1}$ and $C_{2}$, respectively. $C_{1}$ is very small in case of single-turn primary $\mathrm{CT}$, hence can be neglected during the analysis. A widebandwidth transformer can reproduce proportional magnitude and the wave shape of the fast-rising current pulse at the secondary. For the bandwidth analysis, it was assumed that the core is being operated in the linear region.

The magnetising inductance $\left(L_{\mathrm{m}}\right)$ is given by

$$
\begin{aligned}
L_{m} & =\frac{\mu \mathrm{n}^{2} \mathrm{Ac}}{\mathrm{Le}}=\frac{4 \Pi \times 10^{-7} \times 7000 \times 35^{2} \times 244.7 \times 10^{-6}}{152 \times 10^{-3}} \\
& =17.3 \mathrm{mH} .
\end{aligned}
$$

The core resistance $R_{\mathrm{c}}=\rho_{\mathrm{c}} \frac{\mathrm{Le}}{\mathrm{Ae}}=\frac{0.2 \times 152 \times 10^{-3}}{244.7 \times 10^{-6}}$

$$
=124.31 \Omega \text {. }
$$

For the selected core, the total length of the secondary winding is given by

$$
\begin{aligned}
l_{\mathrm{s}} & =2 \mathrm{n}\left(\mathrm{h}+\frac{\mathrm{O} . \mathrm{D} . \mathrm{I} . \mathrm{D} .}{2}\right) \\
& =2 \times 35 \times\left(20+\frac{63-38}{2}\right) \times 10^{-3}=2.275 \mathrm{~m} .
\end{aligned}
$$

Therefore, the dc and low-frequency secondary winding resistance for a copper AWG 24/19/36 wire with resistance per length of $69.16 \Omega / \mathrm{km}$ and resistivity of $1.7 \times 10^{-8} \Omega-\mathrm{m}$ is obtained as $0.15 \Omega$.

$$
\begin{aligned}
& \frac{i_{B}(s)}{\frac{i(s)}{n}}= \\
& \frac{\mathrm{s}\left(\mathrm{s}_{\mathrm{m}}+\mathrm{L}_{\mathrm{m}}\right)}{\left.\mathrm{CR}_{\mathrm{B}} \mathrm{L}_{\mathrm{m}} \mathrm{L}_{2}\right)+\mathrm{s}^{2}\left(\mathrm{CR}_{\mathrm{B}} \mathrm{L}_{\mathrm{m}} \mathrm{R}_{\mathrm{m}}+\mathrm{CR}_{\mathrm{B}} \mathrm{L}_{\mathrm{m}} \mathrm{R}_{2}+\mathrm{L}_{\mathrm{m}} \mathrm{L}_{2}+\mathrm{CR}_{\mathrm{B}} \mathrm{L}_{2} \mathrm{R}_{\mathrm{m}}\right)+\mathrm{s}\left(\mathrm{R}_{2} \mathrm{~L}_{\mathrm{m}}+\mathrm{R}_{\mathrm{B}} \mathrm{L}_{\mathrm{m}}+\mathrm{CR}_{\mathrm{B}} \mathrm{R}_{\mathrm{m}} \mathrm{R}_{2}+\mathrm{R}_{\mathrm{m}} \mathrm{L}_{2}+\mathrm{R}_{\mathrm{m}} \mathrm{L}_{\mathrm{m}}\right)+\left(\mathrm{R}_{\mathrm{m}} \mathrm{R}_{2}+\mathrm{R}_{\mathrm{m}} \mathrm{R}_{\mathrm{B}}\right)}
\end{aligned}
$$




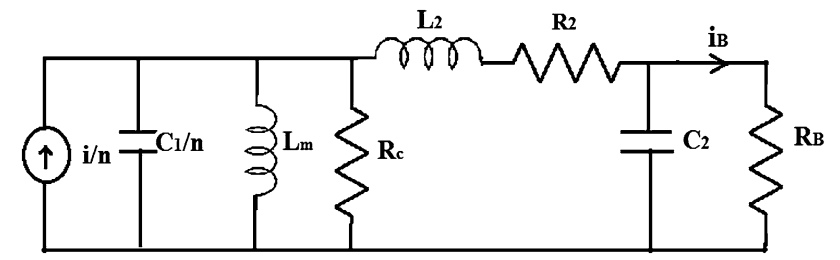

Figure 5. Equivalent circuit model of the current transformer with current source and primary side capacitance reflected to secondary.

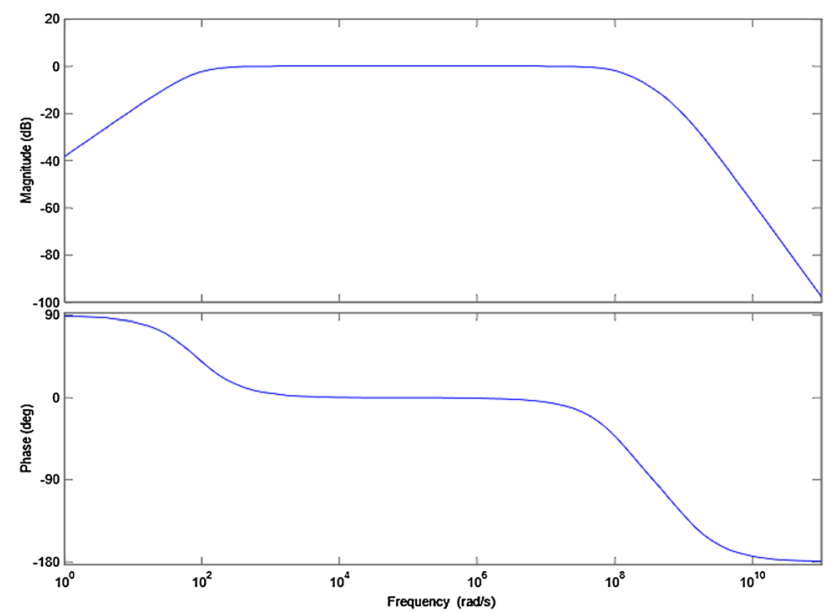

Figure 6. Bode plot of current transformation ratio of the CT (magnitude and phase plot).

The current transformation ratio is arrived at by solving the equivalent circuit model of the CT shown in figure 5. where $i(s)$ and $i_{B}(s)$ are the Laplace transform of the primary current and current flowing through the burden resistance, respectively.

Figure 6 shows the bode plot of the current transformation ratio of the $\mathrm{CT}$ with the following parameters: $L_{\mathrm{m}}=17.3 \mathrm{mH}, R_{\mathrm{m}}=124.31 \Omega, R_{2}=0.1 \Omega$ and measured values of $L_{2}=0.9 \mu \mathrm{H}, C=0.8 \mathrm{nF}$. From the magnitude plot, the lower cut-off frequency is $100 \mathrm{rad} / \mathrm{s}$ i.e. $16 \mathrm{~Hz}$ while the upper cut-off frequency is $100 \mathrm{Mrad} / \mathrm{s}$ i.e. $16 \mathrm{MHz}$. The CT acts as the band pass filter with a wide bandwidth of nearly $16 \mathrm{MHz}$. The unity gain magnitude of the transformation ratio over the operating range of the frequency ensures that the ratio of primary and secondary current is equal to the turns ratio (n) of the CT. The use of high permeability core, reduced parasitic capacitance, low droop and high $i-t$ product ensured reduced good fidelity of the pulsed current signal.

The calibration of the CT has been done with the Pearson current monitor model 110 make having the sensitivity of $0.1 \mathrm{~V} / \mathrm{A}$. Figure 7 shows the measurement of the current signal using Pearson current monitor and HFCT developed having the sensitivity of the 0.037 V/A. The calibration factor of the current measurement was 2.65 between the peak values of the Pearson and the HFCT signals.

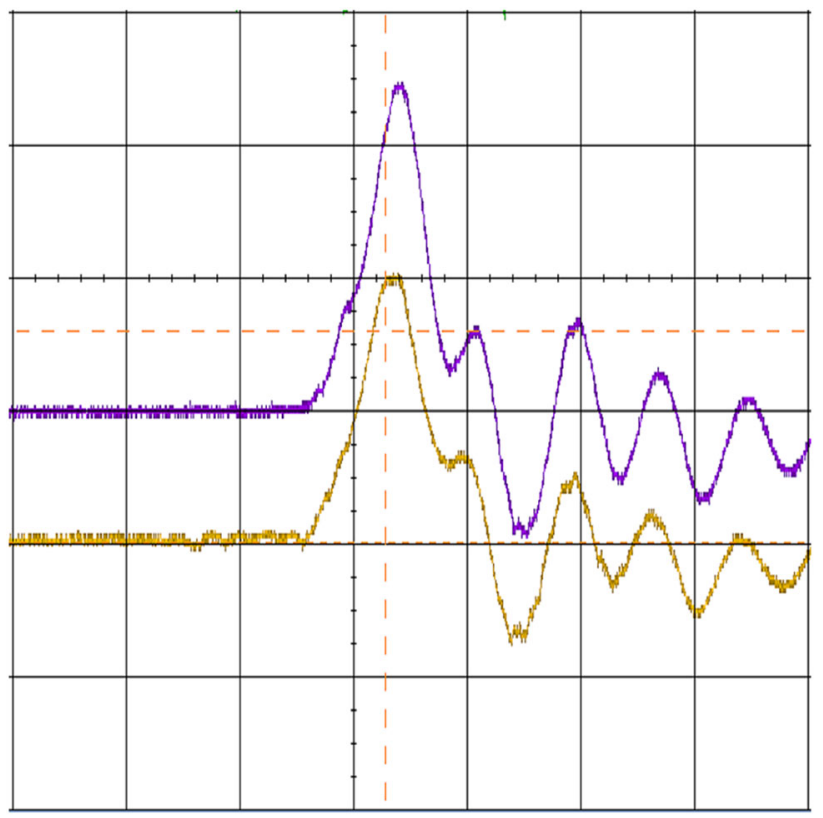

Figure 7. Typical current waveform through the inductor during bad CVL discharge condition. Scale: $\mathrm{X}$ axis $-2 \mu \mathrm{s} / \mathrm{div}$; $\mathrm{Y}$ axis 2 V/div (violet) - current signal measured using Pearson current monitor; $\mathrm{Y}$ axis $-1 \mathrm{~V} / \mathrm{div}$ (yellow) - current signal measured using HFCT.

\subsection{Optoelectronic conversion}

The voltage signal developed across the burden resistance is directly used to passively drive the optical transmitter. The $820 \mathrm{~nm}$ technology based HFBR 1424 from 0400 series manufactured by Avago Technologies is used. The $62.5 \mu \mathrm{m}$ fiber optic cable with FC connectors at both ends formed the fiber optic link. Series resistance $R_{2}$ was placed to limit the current below the maximum permissible forward input current. Figure 8 shows the image of the optoelectronic-based pulsed current sensor.

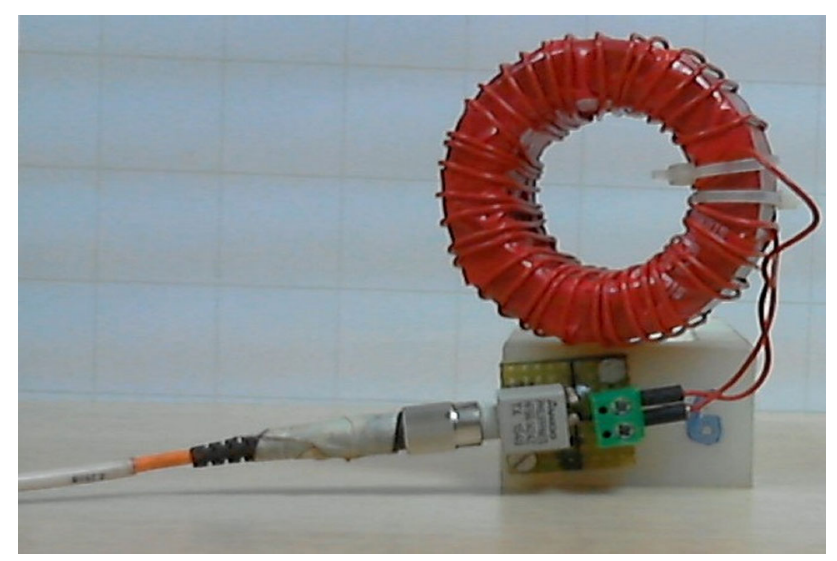

Figure 8. Photo of the optoelectronic-based pulsed current sensor setup. 


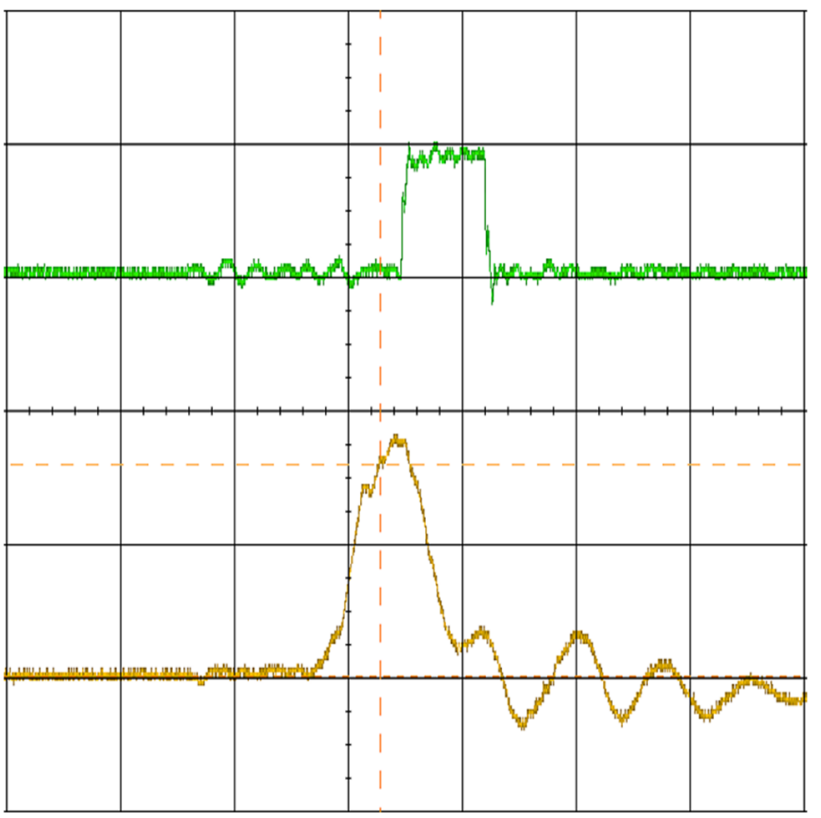

Figure 9. Current waveform through the inductor during bad CVL discharge condition. Scale: $\mathrm{X}$ axis $-2 \mu \mathrm{s} / \mathrm{div}$; $\mathrm{Y}$ axis $1 \mathrm{~V} /$ div (yellow) - current signal measured using HFCT; Y axis $5 \mathrm{~V} / \mathrm{div}$ (green) - TTL output at the HFBR receiver.

The optical receiver HFBR 2422z was used for optical to electrical conversion. The HFBR receiver incorporates an integrated photo IC containing a photo detector and dc amplifier driving an open-collector Schottky output transistor. The external pull-up resistance $R_{3}$ ensures valid TTL logic level at the open collector output available at pin 2 of the HFBR 2422z. The TTL signal will only be generated when the primary inductor current has exceeded the set threshold value. The digital signal thus generated is used to generate a sound alarm indicating poor laser discharge condition. Figure 9 shows the generation of the TTL signal when the output voltage of the HFCT just exceeds threshold $V_{\text {on }}=1.7 \mathrm{~V}$. The digital output is used as the trigger input to the dual-precision monostable multi-vibrator IC 4538. The monostable multi-vibrator IC was operated in a retrigger mode of operation in order to obtain continuous dc voltage at the output. The dc voltage drives the transistor BC 547 into conduction and the buzzer alarm connected in series with the collector gets activated. Thus, the buzzer sounds only when the primary current flowing through the inductor has exceeded the threshold limit of $45 \mathrm{~A}$.

\section{Discussions and conclusions}

The optoelectronic-based pulsed current sensor has been developed to detect the poor discharge condition of the copper vapour laser by sensing the current flowing through the bypass inductor. The current transformer is designed with the sensitivity of the $0.037 \mathrm{~V} / \mathrm{A}$ and wide bandwidth of $100 \mathrm{Mrad} / \mathrm{s}$ to ensure operation of the pulsed current sensor at current threshold crossover of $45 \mathrm{~A}$ peak and high fidelity of the actual current pulse of rise time of $200 \mathrm{~ns}$. The remote signal processing in an EMI-immune environment is possible due to the optical isolation between the high voltage and digital circuitry. The sensing circuit is developed to give an alarm so that the operator can take necessary actions to ensure safe shutdown. The testing of the system is currently underway to ensure no human intervention during such abnormal conditions. The signal obtained at the output of the receiver can be utilised to trigger the automatic trip system, thus bringing the system automatically to the safe shutdown. The automatic trip system consists of the several interlocks connected in AND logic inside the PLC. The output of the AND logic is communicated back to the system using the PLC, thus changing the relay contact, which shuts down the highvoltage system.

This technology can be extended to generate an interlock/ alarm for different high-power-discharge lasers like flash lamp pumped Q-switch solid-state lasers. The pulsed current sensor based on the current transformer (CT) or the Rogowski coil techniques can be used in other pulsed laser systems and discharge current measurements. The current transformer gives higher-output voltage for the same dimension coil as compared to the Rogowski coil provided that the core of the $\mathrm{CT}$ is not saturated. In order to measure high-pulsed current, the required size of the ferrite core would be large in order to avoid the saturation. The same-sized CT can handle higher current if the repetition rate is on the lower side. Hence, depending on the current and pulse repetition rates, the size of the core needs to be finalised. The Rogowski coil-based technique could be used along with the integrator. Since the Rogowski coil consists of air core, the output is immune from saturation. The optoelectronic inter-conversion technique will remain the same irrespective of the technology used to sense the current.

\section{Acknowledgements}

The authors would like to acknowledge the contributions of entire laser group of L\&PTD for their immense guidance, motivation and support.

\section{References}

[1] Xiao C, Zhao L, Asada T, Odendal W G and Wan Wyk J D 2003 An overview of integratable current sensing technologies Conference Record of IEEE Industry Applications Conference 2 (Oct): 1251-1258

[2] Dyer S A 2001 Wiley survey of instrumentation and measurement. New York: Wiley

[3] Limcharoen W and Yutthagowith P 2013 Rogowski coil with an active integrator for measurement of switching impulse 
current. IEEE International Conference on Electrical/Electronics, Computer, Telecommunication and Information Technology (ECTI-CON), pp 1-4

[4] Metwally I A 2010 Self-integrating Rogowski coil for highimpulse current measurement. IEEE Trans. Instrum. Measur. 59(2): 353-360

[5] Bo C, Qiufeng C, Chengqun Y and Yihan Y 2002 Study on new optoelectronic current transformer. In: Proceedings of
International Conference on Power Systems and Technology (PowerCon) 2: 1105-1109

[6] Djokic B 2006 An optically isolated hybrid two stage current transformer for measurements at high voltage. IEEE Trans. Instrum. Measur. 55 (2): 1204-1207

[7] Kondrath N and Kazimierczuk M 2009 Bandwidth of current transformers. IEEE Trans. Instrum. Measur. 58(6): 2008-2016 